\title{
Aspects of Non-linear Optics
}

\author{
D.A.H. Mace and M.J. Adams, Ipswich
}

(Research Laboratories of British Telecom, an EPS Associate Member)

There is considerable current interest in the potential use of non-linear optical effects for optical processing and switching applications in communications systems. For although signals may be transmitted optically along optic fibres, they are normally processed or switched electronically. Replacing this later stage with optical processing using non-linear optics may not only eliminate much complicated circuitry such as detectors, transistors and laser re-transmitters, but also increase the bandwidth of the system and open the way to new 'parallelprocessing' techniques, uniquely suited to optical fibres.

Before discussing non-linear optics, consider the salient features of linear optics. When an electromagnetic wave penetrates into a medium from free space it slows down. This is a consequence of interference with radiation produced within the medium that is phase shifted with the incoming wave. Driving the radiation are the oscillations of the polarization of the internal charge distribution induced by the incoming wave. For a wave of electric field $E$, travelling from free space into a material of susceptibility $\chi$, the induced oscillating polarization $P=\chi E$. Radiation subsequently produced is phase shifted by an amount depending on the difference between the resonance frequency of the charge distribution and the frequency of the incoming wave. Interference follows and results in a cancellation of the incoming primary wave travelling at velocity $c$ and production of a second wave whose phase velocity is $c / n$ where $n$ is the refractive index and is given by $(1+\chi)^{1 / 2}$. In addition, a change of direction occurs according to $\sin \theta / v=$ constant, (Snells law) where $v$ is the velocity of a wave travelling at angle $\theta$ to the normal of the free space/medium interface. Clearly both wave velocity and direction of propagation are affected by the susceptibility of the medium.

To a first approximation, the susceptibility is independent of electric field for most materials. Consequently the polarization is linear in electric field. This is the linear optics regime. The path of an optical wave is therefore not affected by either the intensity of the beam itself or by the presence of other beams; the optical waves cross one another without scattering. Under certain conditions however, the susceptibility is strongly dependent on electric field and the approximation becomes invalid. This is the regime of non-linear optics. To describe this field dependence, the susceptibility is written as a series of the form:

$$
\chi=\chi^{(1)}+\chi^{(2)} E+\chi^{(3)} E^{2}
$$

Clearly manipulation of the path of the beam by its own electric field or by other beams is now possible. This is essential for optical processing.

If the electric field component of a wave in the medium takes the form of $E_{0} \cos \alpha$ where $\alpha=(k z-\omega t)$, (a wave of frequency $\omega$, wavevector $k$, travelling in the $+z$ direction) then the polarization induced is given by:

$P=\chi E=\chi^{(1)} E_{0} \cos \alpha+\chi^{(2)} E_{0}^{2} \cos ^{2} \alpha+$ $\chi^{(3)} E_{0}^{3} \cos ^{3} \alpha+\ldots$

As $\cos ^{2} \alpha=1 / 2(\cos 2 \alpha+1)$, the $\chi^{(2)}$ term describes second harmonic generation and rectification. The $\chi^{(3)}$ term however, describes waves generated at both the same frequency as the incoming wave and three times this frequency, since $\cos ^{3} \alpha=3 / 4 \cos \alpha+1 / 4 \cos 3 \alpha$. For the purposes of optical processing we are not interested in radiation generated at frequencies other than the frequency of the incoming wave. Hence the $\chi^{(2)}$ effects are omitted in this article. This omission is further justified because cumulative build-up of the second harmonic (or third harmonic) does not normally occur as its phase velocity (2 $\omega / k$ ) is not (phase) matched to the natural phase velocity of the medium. The other component described by the $\chi^{(3)}$ term does not diminish however, because it has the same velocity $(\omega / k)$ as the incoming wave which in turn travels with the natural phase velocity of the medium. (The incoming wave slows down to the natural phase velocity of the medium when it enters the medium.) Notice that this phase matched component is very similar to the wave described by the $\chi^{(1)}$ term except $\chi^{(1)}$ is replaced by $3 / 4 \chi^{(3)} E_{0}^{2}$. Consequently the radiation produced by the oscillatory internal charge distribution consists of two com- ponents, phase matched to the incoming wave, one of which is generated according to a susceptibility that is dependent on $E_{0}^{2}$, i.e. the intensity of the incoming wave. The effective susceptibility of the medium is thus

$$
\chi=\chi^{(1)}+3 / 4 \chi^{(3)} E_{0}^{2}
$$

and the refractive index is

$$
n=n_{0}+n_{l} l
$$

where $n_{0}$ is the linear refractive index (dependent on $\left.\chi^{(1)}\right)$, and $n_{1}\left(\mathrm{~cm}^{2} /\right.$ watt $)=$ $12 \pi^{2} \chi^{(3)}$ (esu) $\times 10^{7} / \mathrm{c} / \mathrm{n}^{2}$ with $c$ the velocity of light in $\mathrm{cm} \mathrm{s}^{-1}$. Clearly the path of the refracted wave resulting from interference of the induced radiation is intensity dependent if $\chi^{(3)}$ is large enough. This leads to self-focussing (or defocussing). In general however, the intensity dependent component may be ignored, for the intrinsic $\chi^{(3)}$ of the medium is usually several orders of magnitude smaller than $\chi^{(1)}$. Therefore most optical phenomena may be described using linear optics to a first approximation although exceptions occur if the optical wave transfers energy to the

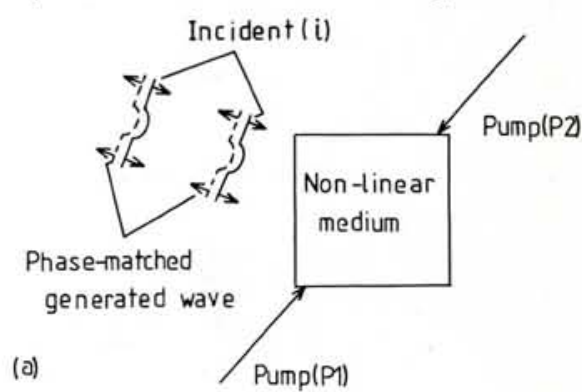
(b)

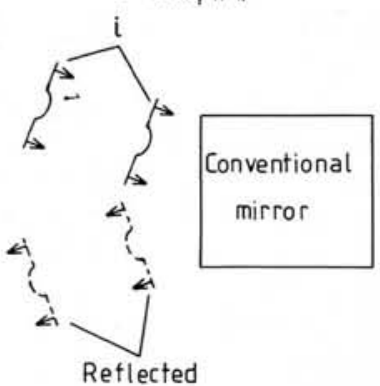

Fig. 1 (a) and (b) - The effect on the wavefront of a wave incident on a non-linear medium pumped by two counter-propagating pump waves, and on a conventional mirror respectively. Note the reversed wavefront and direction of propagation of the phasematched wave generated nonlinearly. It is displaced from the incident wavefronts for illustrative purposes. 
(a)

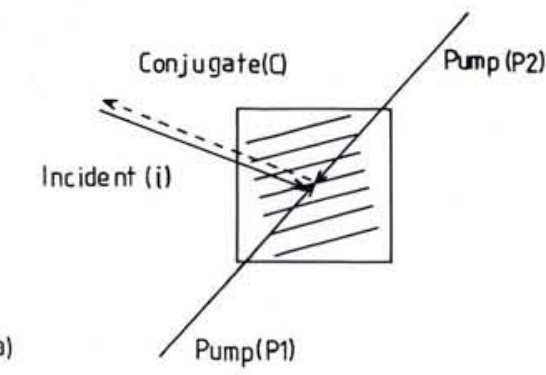

(b)

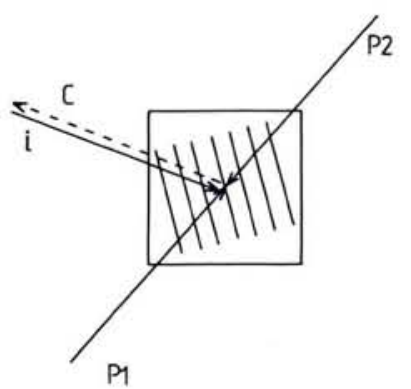

(b)

PI

Fig. 2 (a) and (b) - Phase conjugate wave resulting from scattering of one pump wave by the static refractive index grating formed by the incident wave and the other pump wave. The grating is formed by $P 1$ and $i$ in (a) and $P 2$ and $i$ in (b).

medium, in which case $\chi^{(3)}$ and $\chi^{(2)}$ may increase substantially. This is discussed later.

So far we have only considered the effect of increasing the intensity of the incoming wave itself. Consider now the result of applying two intense counterpropagating waves (P1 and $\mathrm{P} 2$ ) of the same frequency and wavelength as a relatively weak incoming probe wave (i). For convenience let the pump waves travel along the $y$ axis and the probe wave along the positive $x$ axis. The cubic term of the polarisation thus takes the form:

$\chi^{(3)}\left(E_{\mathrm{p}_{1}} \cos (k y-\omega t)+E_{\mathrm{p} 2} \cos (k x+\omega t)\right.$ $\left.+E_{i} \cos (k x-\omega t)\right)^{3}$

Simple algebra reveals that three phase matched waves are generated in the same direction as the probe wave, and one in the opposite direction whose form is:

$$
E_{\mathrm{p} 1} E_{\mathrm{p} 2} E_{i} \cos (k x+\omega t) .
$$

This last wave is particularly interesting as the form of its wavefronts are the reverse of the incoming probe wave. This is illustrated in Fig. 1a. (Note that the pump waves are not drawn orthogonal to the probe wave. This is to indicate that the wavefront reversed wave is produced in the opposite direction to the incoming wave regardless of the orientation of the pump waves.) For comparison, the reflection of a similar probe wave in a normal mirror, where wavefront reversal does not occur is shown in Fig. 1b. The reversal of the wavefront is mathematically equivalent to producing a wave with the conjugate phase. Hence the newly generated phase matched wave is often referred to as the 'phaseconjugate wave'. The origin of this wave may be interpreted by considering the effects of any two of the optical waves on the refractive index of the material and the subsequent scattering of the third wave. The two counter-propagating pump beams cause the refractive index of the medium to 'breathe' everywhere at $2 \omega$. The incoming wave at frequency $\omega$ subsequently scatters off this 'breathing' refractive index to produce waves at frequency $3 \omega$ and $\omega$, although only those oscillating at $\omega$ with the conjugate phase are phase-matched. Alternatively, interference of the first pump wave and the incoming wave results in a static interference pattern. If the amplitude of the pump beam is large enough, this interference pattern creates a static spatial modulation of the refractive index. The second counter-propagating pump beam subsequently Bragg scatters off this refractive index grating to create a wave travelling in the opposite direction to the incoming wave with the conjugate phase (see Fig. 2a). Similarly interference between the second pump beam and the incoming wave is a possible description, with the formation of a different static grating. Once again a phase conjugate wave in the opposite direction to the incoming wave is produced. This is illustrated in Fig. $2 \mathrm{~b}$.

The static gratings are analogous to the grating formed in the photographic emulsion of a hologram. In holography part of a reference wave is directed onto the photographic emulsion while the remaining part is directed onto the object. The wave subsequently reflected off the object then interferes with the reference wave, in the same way as the incoming wave interferes with one of the pump waves to produce a refractive index grating in the emulsion. Subsequent illumination of the developed hologram at a later stage with a reference beam identical to the first, but travelling in the opposite direction, results in a wave scat-

Fig. 3 - Comparison between the reciprocal of the intensity dependent refractive index $n_{1}$ and the associated process time $\tau$ for several bulk semiconductors and AIGaAs/GaAs MQW structures. 1: Islam et al. (1985) using $n_{0}=3.38$ from Broberg et al. (1984); 2: Poole et al. (1984); 3: Chemla et al. (1985); 4: Miller et al. (1981); 5: Garmire et al. (1985) (Theoretical value); 6: West et al. (1985). tered off the grating that is the conjugate replica of the wave reflected off the object. It is travelling in the opposite direction and forms, in holographic terms, a pseudoscopic image where the object was originally. Clearly phase conjugate optics and conventional holography are closely related. This mixing of three waves non-linearly to produce a fourth is often referred to as degenerate (if all the waves oscillate with the same frequency) four wave mixing (DFWM).

\section{Devices}

There are several reasons for choosing semiconductors in favour of other materials for non-linear optical devices in communications systems. First, compatibility with current semiconductor device technology, allows the possibility of hybrid integration with other electronic devices. Second, large increases in $\chi^{(3)}$ are possible for semiconductors under certain conditions. As the susceptibility is a measure of the response of the internal charges to an applied electric field then, not surprisingly, processes which involve changes in the number of charges or their effective mass will alter the susceptibility. If these processes depend upon the intensity of the optical waves (rather than the amplitude), i.e. involve transfer of energy into the medium, they are described by $\chi^{(3)}$. They fall into two categories - resonant and non-resonant transitions.

Resonant transitions (or interband excitations) involve excitation across the bandgap with intense electric fields to create large changes in the carrier population. Several orders of magnitude increase in $\chi^{(3)}$ is possible, although times of the order of ns (electron-hole recombination time) are necessary before the process may be repeated.

Non-resonant transitions involve excitation of the carriers within a band (or sub-band). If the bands are non-parabolic these excitations may result in a change in effective mass of the carriers. However the resulting modification to the susceptibility is usually much

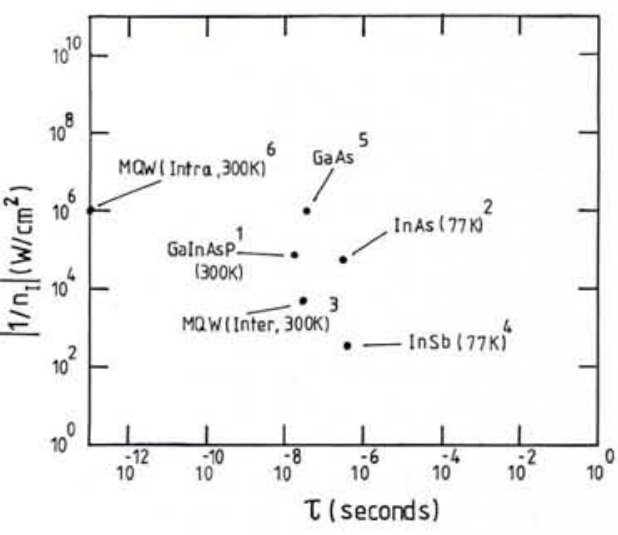


Fig. 4 - Schematic illustration of a semiconductor laser amplifier.

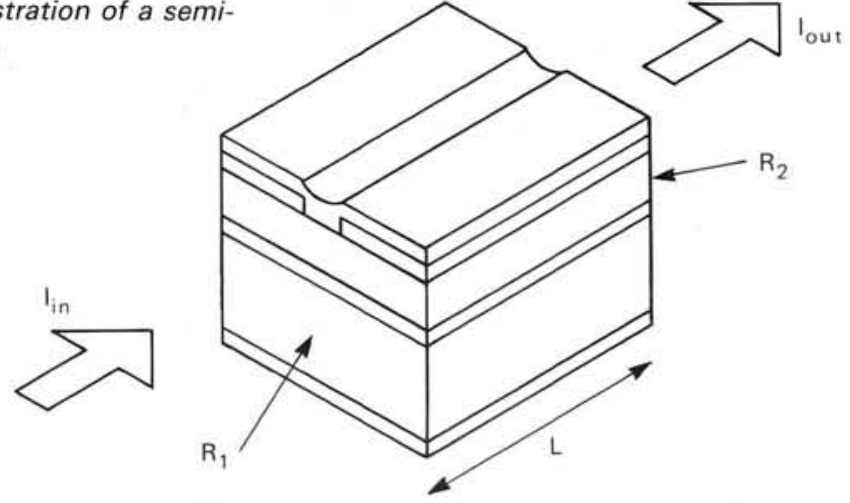

smaller than for resonant transitions, although the process may be repeated more quickly as the relaxation times are short (ps) because the dominant scattering of the electrons is with the phonons. Clearly there is a "trade-off" between the size of $\chi^{(3)}$ and the process time. Examples of this are shown for several bulk semiconductors in Fig. 3, where $1 / n$, is plotted against the associated recombination time $\tau$. Further enhancement of $\chi^{(3)}$ for these processes may be obtained by using low dimensional structures rather than bulk. This is also shown in Fig. 3, for GaAs/GaAlAs, multiquantum well (MQW) structures, where both interband and intrasub-band transitions have been observed.

An example of a device that operates using resonant transitions is the semiconductor laser amplifier. Recall first that such a laser makes use of stimulated emission produced by radiative recombination between the conduction and valence bands of a semiconductor. The emission occurs as a result of high concentrations ( $\cong 10^{18} \mathrm{~cm}^{-3}$ ) of electrons and holes which are injected simultaneously across two heterojunctions into the active region (the 'double heterostructure'). The entire structure is contained within a resonant cavity formed by cleaving the ends of the device along parallel crystal planes (Fig. 4): the semiconductor-air interface produces a reflectivity of about $30 \%$. Lasing threshold is said to occur when the optical gain arising from the stimulated emission is sufficient to balance all the losses from the cavity (due to scattering, absorption, and output radiation).

Now if the device is operated just below the threshold, it will act as an amplifier for an input optical signal of intensity $I_{\text {in }}$, say, as depicted in Fig. 4. For relatively weak input signals, the optical gain available in the active region will be independent of the input optical intensity, and the device operates as a linear amplifier, i.e. the output is a faithful reproduction of the input, but of greater intensity. However, for stronger inputs, the effect of the input signal undergoing amplification is to reduce the free carrier concentration and hence the rate of stimulated emission per unit length of the active region. Since the stimulated emission is related to the refractive index via the dispersion (Kramers-Kronig) relation, there is an associated change of index in the presence of a sufficiently strong optical input. The resultant change of phase in the Fabry-Perot cavity can give rise to strongly non-linear output-input characteristics and to optical bistability $(\mathrm{OB})$. For low input signals, the spectral response of the cavity is a "comb of" longitudinal modes occurring at resonant wavelengths corresponding to single-pass phase periods of $\pi$. However, for higher values of optical input, the extra dependence of phase on optical intensity noted above causes departures from the usual FabryPerot mode spectrum.

To understand the cavity effects in more detail, consider the spectral response shown in Fig. 5 for three values of input optical intensity $l_{\text {in }}$. The figure shows the normalised average intensity in the cavity $\left(I / I_{\mathrm{av}} / \mathrm{S}\right)$ as a function of single-pass phase change $\phi$ for an amplifier at $95 \%$ of lasing threshold.
The quantity $I_{S}$ is a scaling intensity whose numerical value will be given below for a device of current interest. The phase $\phi$ at low input levels is determined by the input wavelength with respect to that of a Fabry-Perot resonance, and is independent of $I_{\mathrm{av}}$. However, for a stronger input, as a consequence of internal intensity-dependent phase effects, the effective phase that the signal sees is different from $\phi$. In fact it transpires that this effective phase is approximately linearly dependent on $I_{\text {av }}$. Thus values of $I_{\mathrm{av}}$ close to the peak of the resonance will induce larger phase shifts than those further away from the peak, resulting in the spectral response shown in Fig. 5. The vertical line marked on the figure at $\phi=-0.1 \pi$ intersects the curve for $I_{\text {in }} / I_{S}=5 \times 10^{-4}$ in three points. Hence an input wavelength corresponding to this $\phi$ will give OB: the upper and lower intersections turn out to be stable solutions, whilst the intermediate one is unstable. To get an idea of the input power levels required, we note that for a $1.5 \mu \mathrm{m}$ amplifier the scaling intensity $I_{S}$ is about $8 \times 10^{5} \mathrm{~W} / \mathrm{cm}^{2}$. The minimum scaled input to give $\mathrm{OB}$ on Fig. 5 is about $10^{-4}$, and it follows that for an amplifier whose active area is about 1 $\mu \mathrm{m}^{2}$ a coupled power of around $1 \mu \mathrm{W}$ should be sufficient to observe OB. This has recently been confirmed by experimental observations on amplifiers at 1.3 $\mu \mathrm{m}$ and $1.5 \mu \mathrm{m}$ operating wavelength. The switching speed is found to be limited by the electron-hole recombination time and is therefore usually in the order of a few nanoseconds.

Apart from the dispersive bistability discussed above, two other forms of $\mathrm{OB}$ in laser structures have been reported, namely absorptive and modal bistability. In absorptive $\mathrm{OB}$ a laser structure is used

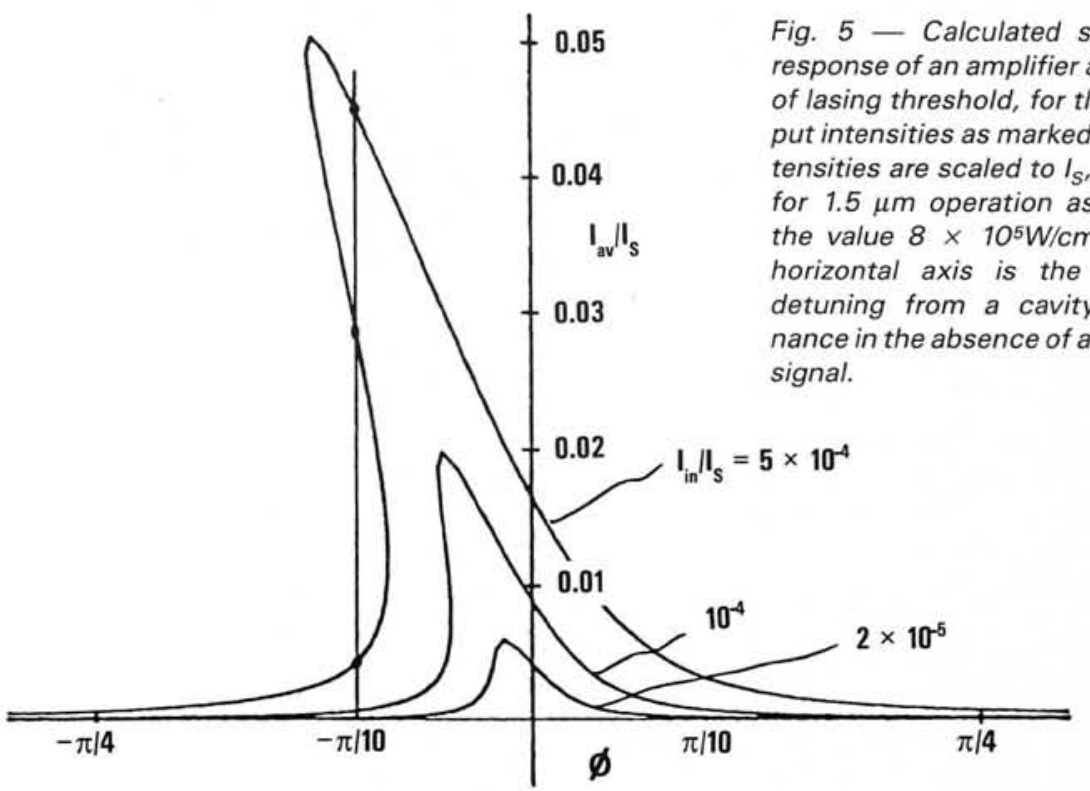


which incorporates a region containing a saturable absorber in series with the active emitting region, both sections lying within the laser cavity. For the case of low optical inputs, the active region of the laser acts as a linear amplifier, but the absorbing region ensures that the optical output is rather low. At somewhat higher input signals, however, the absorbing region is bleached due to saturation of the absorption, and the optical output switches to a high state. The power levels at which the device switches can be controlled to some extent by the currents applied to the absorbing and amplifying regions. A recent version of this structure has been employed as a memory switch at room temperature triggered by an optical pulse of $9-12 \mu \mathrm{W}$ with 4-5ns delay time.

Modal bistability has been observed in lasers where a special twin-stripe contact is used (compare with the single stripe contact for the laser illustrated in Fig. 4). Two parallel stripe contacts are made along the length of the laser cavity, and it is found that under this twinstripe structure there are two distinct lateral modes associated with different waveguiding mechanisms. Switching between these two modes has been achieved optically with an input power of around $1.2 \mathrm{~mW}$. The fastest time to switch between these modes so far observed is 800 ps, but the true limit may be well below this value since carrier distributions corresponding to the two modes are virtually identical and thus carrier lifetime and diffusion times are not expected to limit the switching speed.

Apart from $\mathrm{OB}$ in laser structures, bistable effects in passive semiconductors have been the subject of intense interest. To make a direct comparison with the laser structures, we shall confine attention to devices for which the plot of optical output power versus optical in put power shows hysteresis. Furthermore, we shall be interested in roomtemperature operation only, and concentrate on reported devices having the lowest power required in switch-up and the shortest response time (usually for switch-down, since this is normally the limiting time constant). It is convenient to present the results on a plot of power versus time, as in Fig. 6: the broken lines on this plot represent contours of constant switching energy. The data for the three types of laser bistability discussed above are presented on the figure. The remaining results plotted are those for $\mathrm{ZnS}$ and $\mathrm{ZnSe}$ passive Fabry-Perot etalons, the MOW etalon mentioned above, and the SEED (Self-Electrooptic Effect
Device) - a hybrid device which uses an MOW modulator/detector with an electrical circuit to provide feedback and hence give bistability. Note that electrical as well as optical powers are given for lasers and SEEDs.

Confining our attention to laser amplifiers, SEEDs, and dispersive MOW etalons, from Fig. 6 we may draw the following conclusions:

1) The best optical energy requirements so far achieved are, respectively, 1fJ, $1 \mathrm{~nJ}$, and 100pJ.

2) The best electrical energy requirements for amplifiers and SEEDs achieved are $40 p J$ and $4 n J$, respectively.

Amplifiers as bistable devices have a number of other advantages, however, which include (i) ready availability and compatibility with optical communication systems, and (ii) inherent optical gain so that the ouput from a device can be used to drive subsequent devices. From these considerations and the points made by Fig. 6 , we may anticipate a range of applications for bistable amplifiers in performing optical logic and switching functions.

We wish to acknowledge the contribution of M.G. Burt in the preparation of this article. The work was partially supported by the Joint Opto-Electronic Research Scheme.

\section{FURTHER READING}

Bloembergen J., Nonlinear Optics (Benjamin, New York) 1965.

Adams M.J., "A Tentative Assessment of Semiconductor Laser Optical Bistability" Int. J. Electronics 60 (1986) 123.

Fisher R.A., Optical Phase Conjugation (Academic Press) 1983.

Gibbs H.M., Optical Bistability; Controlling Light with Light (Academic Press) 1985.

Midwinter J.E., 'Light' Electronics, Myth or Reality? IEE Proceedings J. 132 (1985) 6, 371.

Shen Y.R., The Principles of Nonlinear Optics (Wiley, New York) 1984.

Yariv A., Introduction to Optical Electronics (Holt, New York) 1971.

\section{REFERENCES}

Broberg B. and Lindgren S., J. App. Phys. 55 (1984) 9, 3376.

Chemla D.S., Miller D.A.B. and Smith P.W., Opt. Eng. 24 (1985) 4, 556.

Garmire E., Opt. Eng. 24 (1985) 4, 575.

Islam M.N., Ippen E.P., Burkhardt E.G. and Bridges T.J., Appl. Phys. Lett. 47 (1985) 10, 1042.

Miller D.A.B., Seaton C.T., Prise M.E. and Smith S.D., Phys. Rev. Lett. 47 (1981) 3, 197. Miller D.A.B., Smith S.D. and Seaton C.T., IEEE Quantum Electronics QE-17 (1981) 3, 312.

Poole C.D. and Garmire E., Opt. Lett. 9 (1985) 8, 356.

West L.C. and Eglash S.J., Appl. Phys. Lett. 46 (1985) 12, 1156.

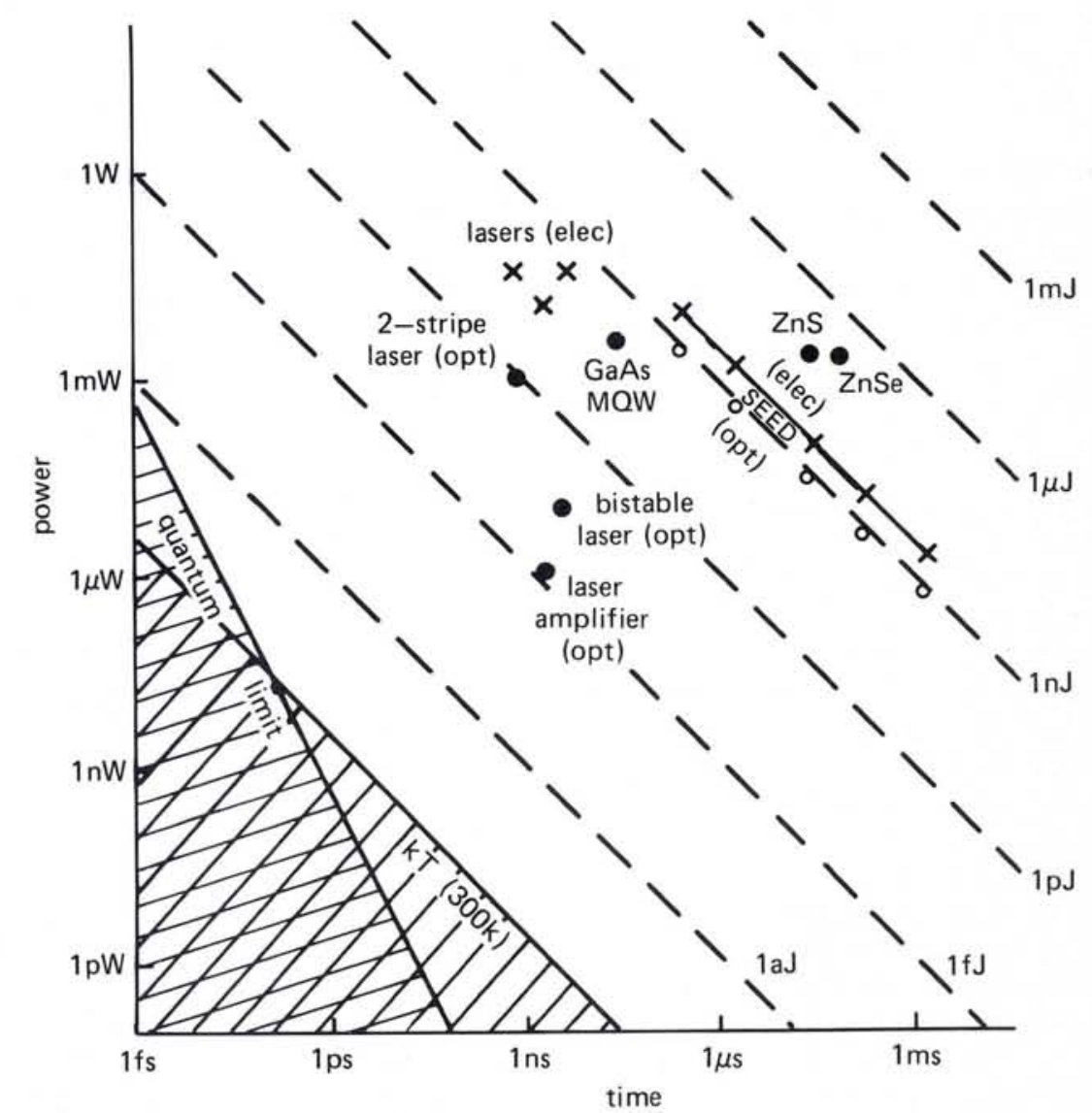

Fig. 6 - Power versus time for best reported bistability results in semiconductors. (For details see Adams M.J. (1986) in 'further reading'). 